\title{
Fabrication of conductive and transparent single-walled carbon nanotube films with PEDOT
}

\author{
Hyungduk Yun ${ }^{1}$, Jong Hun $\mathrm{Han}^{2}$ and Sanghyo Kim ${ }^{1}$ \\ In this paper, the fabrication of highly conductive and transparent films of single-walled carbon nanotubes (SWCNTs) and \\ poly(3,4-ethylenedioxythiophene) (PEDOT) on top of polyethylene terephthalate film (PET) substrates is demonstrated using \\ electropolymerization without sacrificing transparency. A sheet resistance of $250 \Omega \mathrm{sq}^{-1}$ is achieved with a transparency of \\ $93 \%$ at a $550 \mathrm{~nm}$ wavelength. The enhancement in the conductivity was attributed to the ability to bridge the gaps between the \\ individual carbon nanotubes with the help of PEDOT, which increases the number of electric tube-tube junction contacts within \\ the SWCNT network.
}

Polymer Journal (2012) 44, 1238-1243; doi:10.1038/pj.2012.87; published online 23 May 2012

Keywords: electropolymerization; poly(3,4-ethylenedioxythiophene); single-walled carbon nanotube; 4-dodecylbenzenesulfonic acid

\section{INTRODUCTION}

Indium tin oxide (ITO) is one of the most widely used materials in electronics. High conductivity and transparency are unique properties of ITO. ${ }^{1}$ For the past decades, most of the electronics that require display panels have been fabricated by using ITO; however, this material has some disadvantages and limitations. A lack of resources, the high cost of patterning on desired substrates, and poor mechanical properties are the main factors that prevent the application of ITO in many areas. ${ }^{2,3}$ Different approaches have been investigated for producing transparent and conductive layers without using ITO. For example, single-walled carbon nanotubes (SWCNTs) have been used to fabricate conductive and transparent layers. ${ }^{4,5}$ In SWCNTs, electrons flow 10 times faster than in silicon. This material also carries 100 times the current and dissipates heat 20 times more efficiently. Transistors fabricated using SWCNTs can also amplify current 20 times better than conventional silicon-based transistors. SWCNTs have a broad range of sheet resistance values $\left(10-10^{7} \Omega \mathrm{sq}^{-1}\right.$.), excellent transparency, good adhesion, good chemical resistance and good flexibility ${ }^{6-13}$ However, the trade-off between conductivity and transparency still remains a problem for SWCNT applications. Among numerous approaches, poly(3,4ethylenedioxythiophene) (PEDOT) has been added to SWCNT films to enhance the conductivity. ${ }^{14-19}$ PEDOT is one of the most popular conducting polymers because of its high conductivity and transparency in thin oxidized films. This material also exhibits environmental stability. ${ }^{20,21}$ After the first synthesis of PEDOT in the 1990s by Heywnag and Jonas, ${ }^{22}$ many studies focused on improving its insolubility in organic solvents to extend the scope of its application. Surfactants, such as sodium dodecyl sulfate and dodecyl benzene sulfonic acid (DBSA), were used to solve this problem. The organic surfactants allow for the dissolution of the 3,4-ethylenedioxythiphene (EDOT) monomer in aqueous solutions and also act as dopants to enhance the conductivity. ${ }^{23}$ The addition of surfactant to PEDOT has been studied by many groups to facilitate the chemical and electrical oxidation of PEDOT. ${ }^{24}$ Herein, we describe how a spraying method was used to fabricate SWCNT films on top of polyethylene terephthalate (PET), and the EDOT monomer was easily polymerized on top of the SWCNT film by electropolymerization to enhance the conductivity without sacrificing the transparency.

\section{EXPERIMENTAL PROCEDURE}

Materials

SWCNTs were purchased from Nano Solution Co. Ltd in (Jeonju, Korea). An air compressor was purchased from Anset IWATA Corporation (Seoul, Korea). 3,4-Ethylenedioxythiophene and 4-DBSA were purchased from Aldrich (Kyungkido, Korea). The reagents were used as received without further purification. SWCNTs used in the experiment have an average diameter of $1.4-1.7 \mathrm{~nm}$ and a length of $5-20 \mu \mathrm{m}$.

\section{Preparation of SWCNT film}

SWCNT solutions were added to small centrifuge tubes and centrifuged at a speed of 13000 r.p.m. for $90 \mathrm{~min}$ to separate any undesired particles and surfactants. The supernatant containing these materials was removed using a pipette. Leaving a specific amount of SWCNT solution in the microtube, $130 \mu \mathrm{l}$ of distilled water was added to the microtube for dilution, and the SWCNT solution was re-dispersed using a vortex device. Next, the solutions were sprayed on top of a PET substrate using a spray gun, and the resulting film was immediately dried on a hot plate at $70^{\circ} \mathrm{C}$. To remove any remaining surfactant from the SWCNT film, the film was washed with distilled water. Lastly, the fabricated SWCNT film was annealed in an oven at $90^{\circ} \mathrm{C}$ for $1 \mathrm{~h}$.

${ }^{1}$ Department of Bionanotechnology, Gachon University, Seoul, South Korea and 2Energy/Nano materials Research Center, Korea Electronics Technology Institute, Seoul, South Korea

Correspondence: Dr S Kim, Department of Bionanotechnology, Gachon University, San 65, Bokjeong-dong, Sujeong-gu, Seongnam-si, Seoul, 461701 Gyeonggi-do, South Korea. E-mail: samkim@gachon.ac.kr

Received 7 December 2011; revised 18 February 2012; accepted 25 March 2012; published online 23 May 2012 


\section{Preparation of PEDOT-SWCNT film}

An aqueous micellar dispersion was prepared by introducing a specific amount of DBSA in $28 \mathrm{ml}$ of distilled water to a vial and stirring the dispersion with a magnetic stirrer for $1 \mathrm{~h}$. For the DBSA concentration study, five different quantities $(0.001,0.009,0.01,0.02$, and $0.07 \mathrm{~g})$ of DBSA were tested. After DBSA was fully mixed with the distilled water, a specific amount of EDOT monomer was added to the DBSA solution and mixed vigorously using a magnetic stirrer for $1 \mathrm{~h}$. Similarly, three different quantities of EDOT monomer $(0.01,0.03$, and $0.07 \mathrm{~g})$ were used to study the effect of EDOT monomer concentration. Using a specifically designed stage, the SWCNT film was fixed under the stage, and $1000 \mu \mathrm{l}$ of DBSA-EDOT complex solution was poured inside a container that is within the stage. The SWCNT film was used as the anode, and a steel rod was used as the cathode. A constant voltage of $1.2 \mathrm{~V}$ was applied to begin the electropolymerization, with reaction times of 1,2 or $3 \mathrm{~min}$.

\section{Characterization}

Scanning electron microscopy (SEM) studies were performed on SWCNT film and PEDOT-SWCNT film by using a JEOL JSM-750F SEM (JEOL, Seoul, Korea). Transmission electron microscopy was used to observe the presence of PEDOT in the SWCNT networks by using a FEI Titan 80-300 high-resolution transmission electron microscopy from FEI Company (Hillsboro, OR, USA). To monitor the change in the Raman spectra for the as-prepared SWCNT and PEDOT-SWCNT networks, a Ramboss 500i spectrometer was used with an excitation energy of $1.96 \mathrm{eV}(632.8 \mathrm{~nm}$, Ar laser). A four-probe apparatus of CMT-SERIES from Changmin Tech Co. Ltd (Kyunggido, Korea). was used to measure the sheet resistance of SWCNT and PEDOT-SWCNT films. Transparency of SWCNT and PEDOT-SWCNT films was measured using an ultraviolet spectrophotometer at the wavelength of $550 \mathrm{~nm}$.

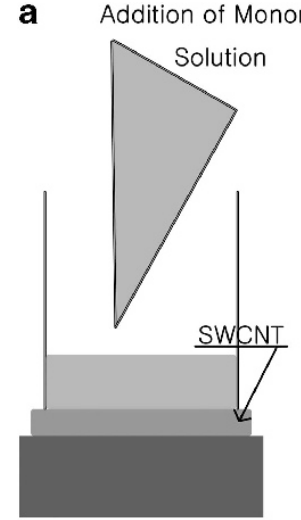

PET Substrate

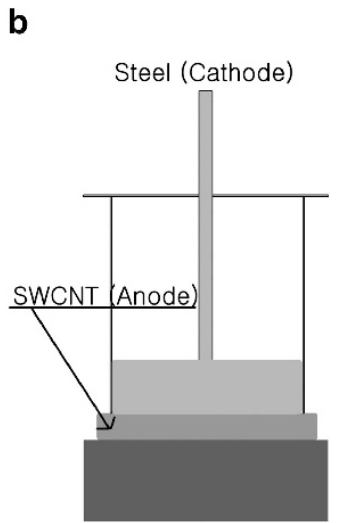

PET Substrate
Figure 1 Schematic diagram of electropolymerization process: (a) installation of electropolymerization stage on top of the single-walled carbon nanotubes film; (b) electropolymerization of poly(3,4-ethylenedioxythiophene) on top of the SWCNT film.

\section{RESULTS AND DISCUSSION}

\section{Electropolymerization stage}

PEDOT can only be synthesized under anodic conditions, which allow it to undergo an electropolymerization. ${ }^{22}$ A specially designed stage was built wherein the SWCNT film served as the anode and a steel rod served as the cathode. Figures 1 and 2 provide a schematic of the electropolymerization stage. To afford a homogeneous polymerization of PEDOT on top of the SWCNT film, the anode electrodes were placed on all four sides of the SWCNT film. In this experiment, two types of anode materials were examined. An aluminum electrode was first used for the electropolymerization on top of the SWCNT film. However, the aluminum electrode failed to transfer a constant voltage from the power supply to the SWCNT film. Aluminum contains a lot of oxide layers in comparison with copper, indium or gold. The oxide layers can act as insulators and disrupt the voltage transfer. When the aluminum electrode was tested, the change in the sheet resistance upon electropolymerization was not consistent. However, when a copper electrode was used consistent data were obtained for the electropolymerized film.

\section{Morphology of SWCNT}

A significant decrease in the sheet resistance was observed after treating the SWCNT film with distilled water due to the removal of the surfactant; however, a representative SEM image of the SWCNT shown in Figure 3 reveals that the surfactant is still present within the SWCNT network. Although surfactant was washed away with distilled water after spraying, the imbedded surfactant was not removed completely. Acid can be used to achieve the complete removal of the surfactant, but there are reports showing that the strong acid damages the surface of the PET substrate. In addition, acid-treated electrodes are difficult to produce on a commercial scale. ${ }^{7}$ Thus, the acid treatment was not performed in this study. The sprayed SWCNT were randomly aligned and exhibited an isotropic structure. Owing to this lack of alignment, a large number of voids between the SWCNTs were also observed.

\section{Morphology of PEDOT-SWCNT}

The synthesis of the PEDOT can be easily observed by the naked eye due to the color change from light blue to dark blue. SEM images of PEDOT-SWCNT are shown in Figure 4. Instead of SWCNTs with sharp edges, the PEDOT-SWCNT film contains SWCNTs with softer edges due to the charging effect. It is likely that the charging effect occurs because there are two different types of material (PEDOT and SWCNTs) that possess different conductivities. By varying the reaction time from $1 \mathrm{~min}$ to $5 \mathrm{~min}$, a significant change in the

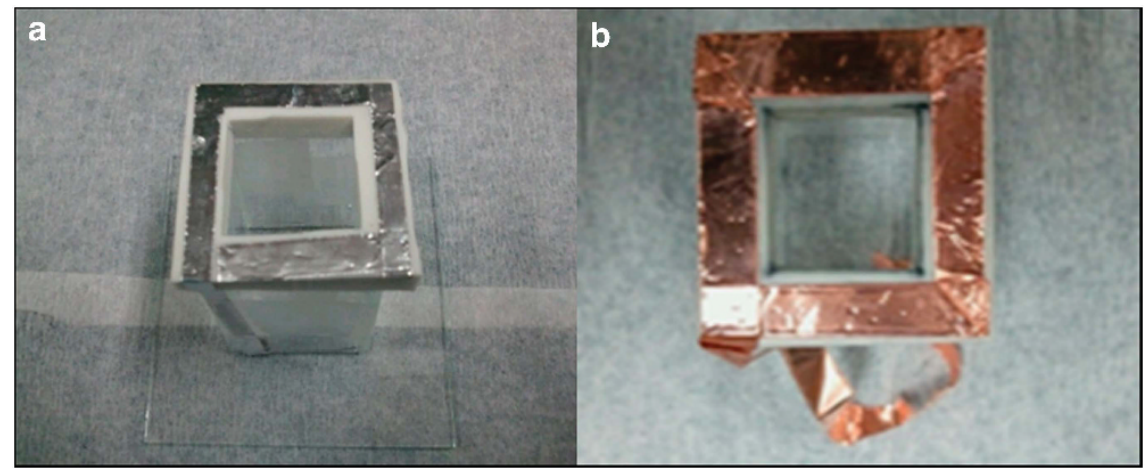

Figure 2 Electropolymerization stage with two different electrodes: (a) aluminum electrode; (b) copper electrode. 
morphology was observed by both the naked eye and SEM. As the reaction time increased, more PEDOT was coated on top of the SWCNT film, such that the fully electropolymerized PEDOT covered

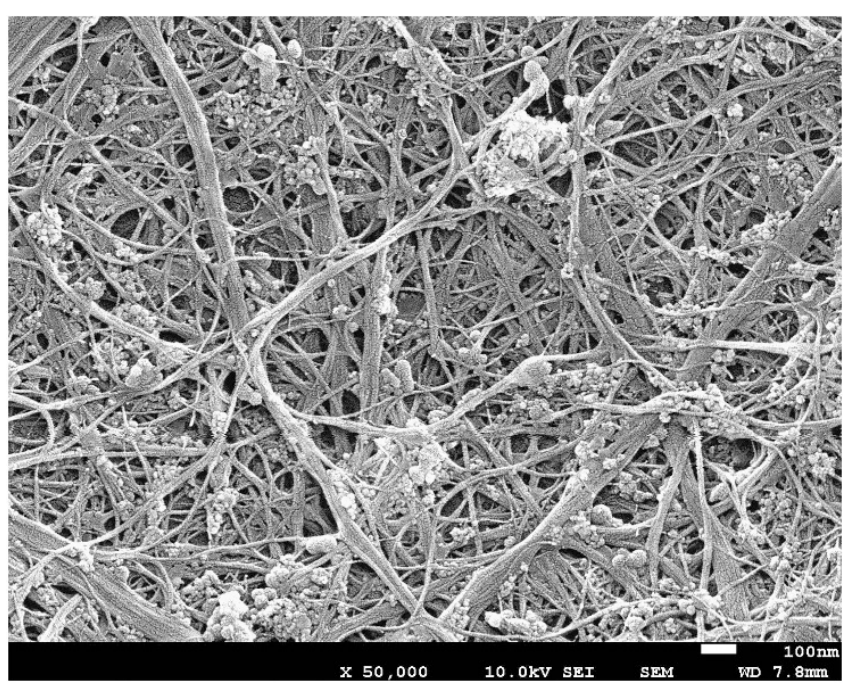

Figure 3 Scanning electron microscopy image of spray-coated SWCNT on a polyethylene terephthalate substrate. most of the voids that were created during the spraying of the SWCNT solution on the PET substrate. PEDOT-SWCNT networks were also observed by transmission electron microscopy. By examining the contrast and color differences between SWCNT and its surrounding background, the PEDOT coating of the SWCNT networks is easily observed, as shown in Figure 5. The morphology of the PEDOT-coated SWCNT appears thicker than that of the pristine SWCNT network. As the majority of the electrical conductivity loss comes from junction resistance, which is mainly due to the imperfect contact between each SWCNT, thicker nanotubes will contribute to a larger surface area for each nanotube's junction, resulting in a decrease in the sheet resistance. After a 1 min electropolymerization of PEDOT on the SWCNT networks, there was no significant effect on the transparency, and the FE-SEM images of PEDOT-SWCNT networks reveal numerous voids formed between each SWCNT. The same morphology was observed by transmission electron microscopy, which displayed a slight coating of PEDOT on the SWCNT network. These results indicate that the electropolymerization of PEDOT on the SWCNT networks for $1 \mathrm{~min}$ did not affect the transparency of the pristine SWCNTs.

\section{Sheet resistance and transparency changes for SWCNT and PEDOT-SWCNT films}

Figure 7 shows the transmission spectra of SWCNT films with different sheet resistances. As mentioned earlier, the trade-off between
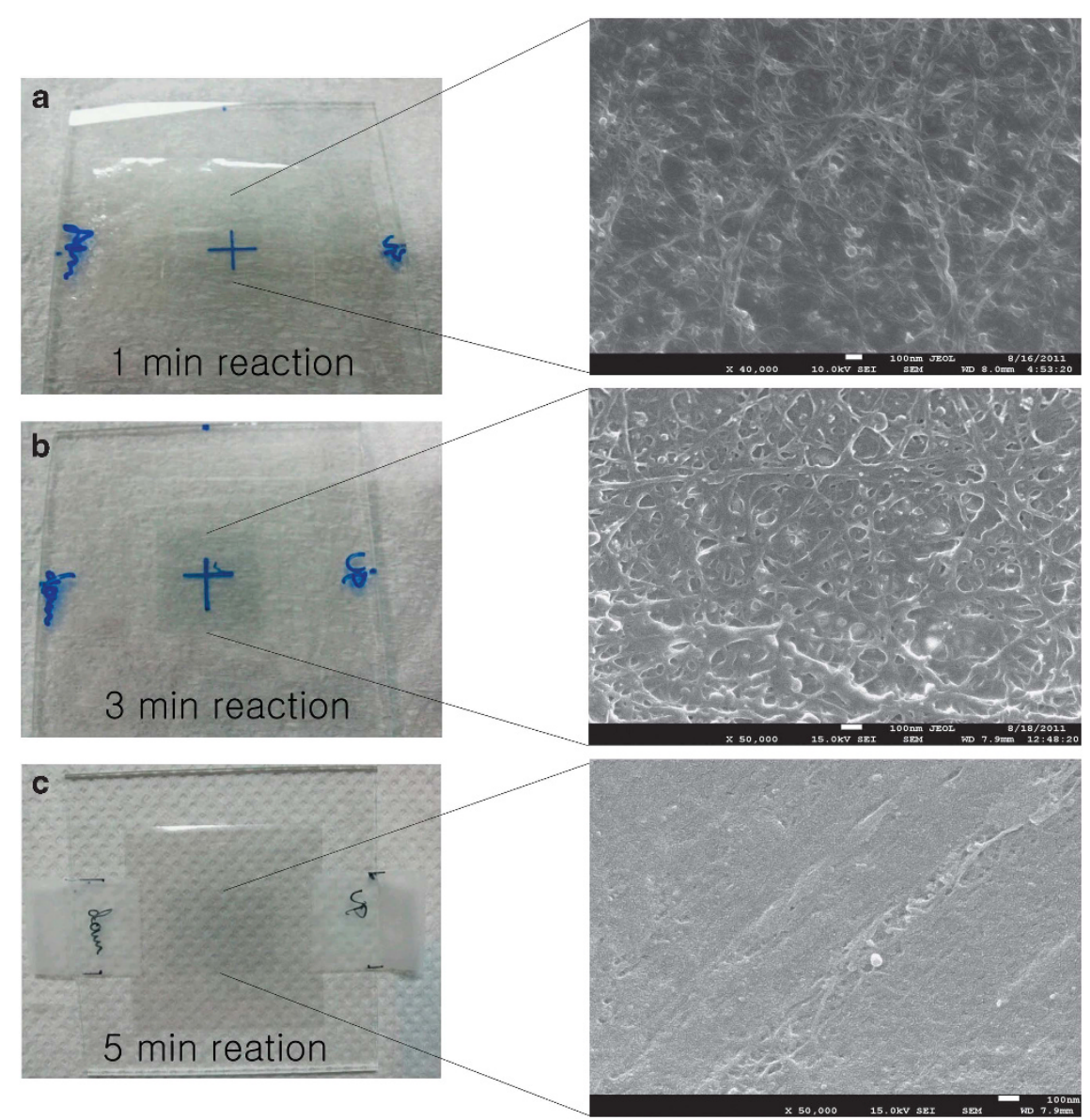

Figure 4 Scanning electron microscopy image of PEDOT-SWCNT (poly(3,4-ethylenedioxythiophene)-single-walled carbon nanotube) film: (a) PEDOT electropolymerized for $1 \mathrm{~min}$; (b) PEDOT electropolymerized for $3 \mathrm{~min}$; (c) electropolymerized for $5 \mathrm{~min}$. 

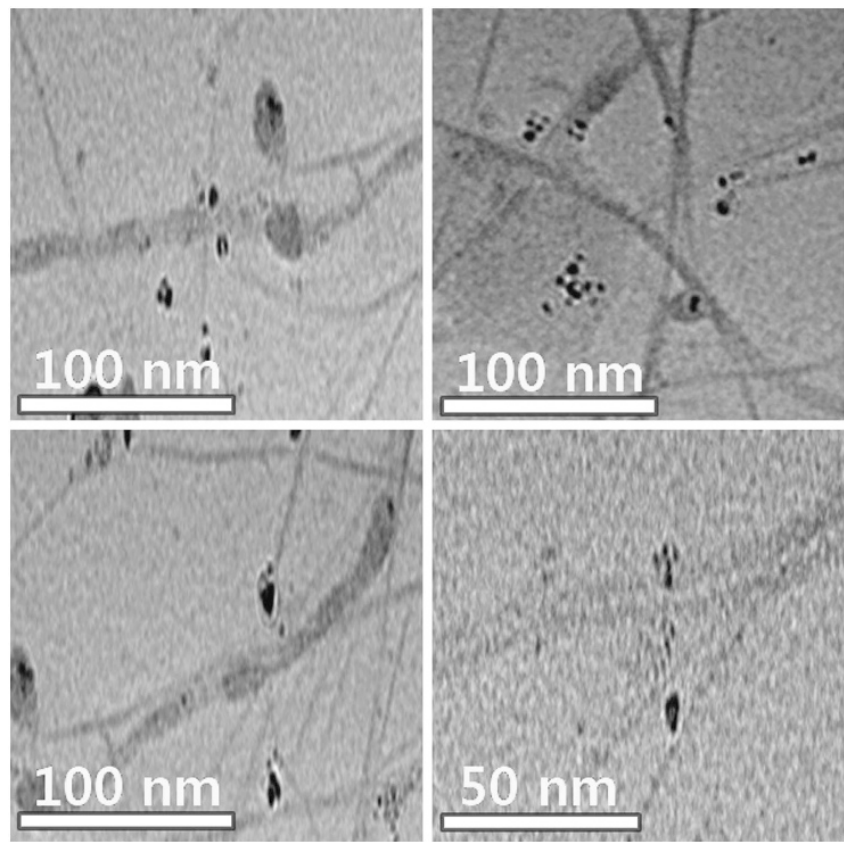

Figure 5 Transmission electron microscopy image of PEDOT-SWCNT (poly(3,4-ethylenedioxythiophene)-single-walled carbon nanotube) film electropolymerized for $1 \mathrm{~min}$.

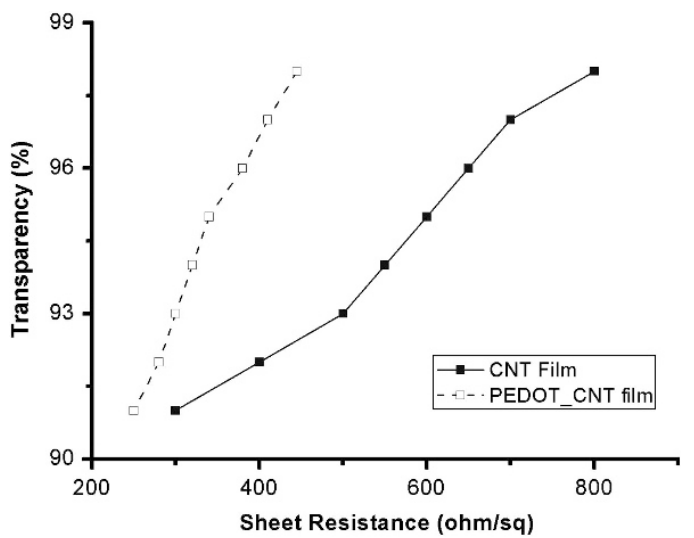

Figure 6 Transparency vs sheet resistance of single-walled carbon nanotube (SWCNT) and poly(3,4-ethylenedioxythiophene)-SWCNT films.

sheet resistance and transparency is clearly observed. An increase in the entanglement and concentration of SWCNT on the substrate decreases the transparency. Figure 6 compares the conductivity and transparency of SWCNT films with those of the PEDOT-SWCNT films. When PEDOT was polymerized on top of SWCNTs, the sheet resistance decreased without sacrificing the transparency. According to the percolation theory, the conductivity of SWCNT depends on three factors: the conductivity of SWCNTs, the concentration of SWCNTs in a particular area and the percolation threshold. ${ }^{6}$ Many studies have confirmed the high conductivity of SWCNTs; thus, the only remaining concern is the percolation threshold. In SWCNTs, the percolation threshold could be interpreted as the capability of free carriers to cross over between each SWCNT in the film. In order for free carriers to be able to move easily and cross over to adjacent SWCNTs, the number of voids must be decreased, which will increase

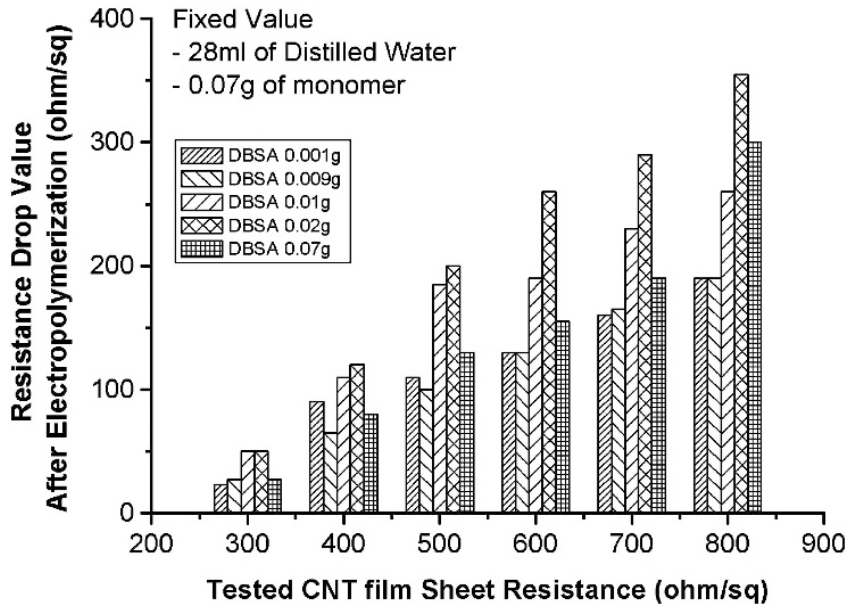

Figure 7 Decrease in the sheet resistance upon electropolymerization using different amounts of dodecyl benzene sulfonic acid (DBSA): DBSA (0.001, $0.009,0.01,0.02$ and $0.07 \mathrm{~g}$.

the points of contact between each SWCNT. Because of the numerous voids presents within the SWCNT network, the voids can disrupt the free carriers from crossing over to adjacent SWCNTs. The physical shape of the nanotubes also makes it difficult to have immaculate contact between each nanotube. The free carriers that succeed in crossing over to each nanotube are directly related to the overall conductivity of the SWCNT film. Thus, by filling in the voids between the SWCNTs with PEDOT, the conductivity of the SWCNT film is expected to increase. Although the PEDOT-SWCNT film prepared using short reaction times does not cover all of the voids between the SWCNTs, an increase in the surface area of PEDOT-SWCNT enhances the degree of contact between each SWCNT and contributes to an increase in the conductivity.

\section{Changes in the sheet resistance due to varying amounts of DBSA} and EDOT

Many studies have shown that the concentration of surfactants such as DBSA or sodium dodecyl sulfate also affects the overall conductivity of the PEDOT due to the doping effect. ${ }^{23}$ In this experiment, five different concentrations of DBSA were tested to analyze the change in conductivity. Figure 7 provides a comparison of the different DBSA concentrations used in the electropolymerizations. As can be observed in the plot, when the amount of DBSA decreased from 0.07 to $0.02 \mathrm{~g}$, the sheet resistance also decreased. However, when the amount of DBSA decreased from 0.02 to $0.001 \mathrm{~g}$, the sheet resistance increased. The decrease in the sheet resistance for films with DBSA content above $0.02 \mathrm{~g}$ is possibly due to the use of excess surfactant. Under these conditions, the surfactant did not act as a dopant, but instead, as an insulating complex. The decrease in the sheet resistance with DBSA content below $0.01 \mathrm{~g}$ might be due to the insufficient doping of the system. ${ }^{24}$ Different quantities of the EDOT monomer were also tested to investigate its influence on the sheet resistance of SWCNT films during electropolymerization. The EDOT monomer content was varied from 0.01 to 0.03 to $0.07 \mathrm{~g}$. Figure 8 illustrates the change in the sheet resistance with the use of different amounts of EDOT monomer. When $0.07 \mathrm{~g}$ of EDOT monomer was used for the electropolymerization, the sheet resistance decreased significantly. In contrast, when the amount of EDOT monomer decreased, the decrease in sheet resistance after electropolymerization 


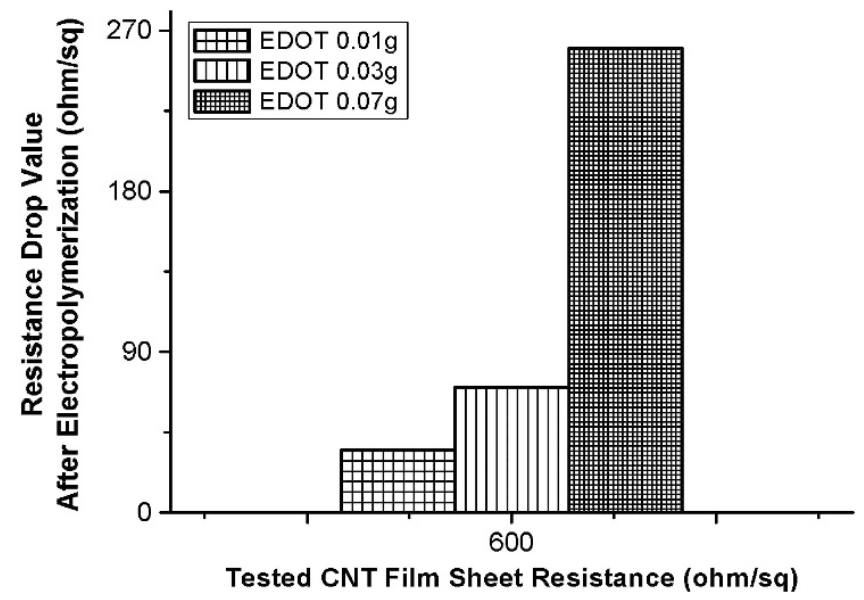

Figure 8 Decrease in sheet resistance upon electropolymerization using different amounts of 3,4-ethylenedioxythiphene (EDOT) monomer: EDOT monomer $(0.01,0.03$ and 0.07$)$.

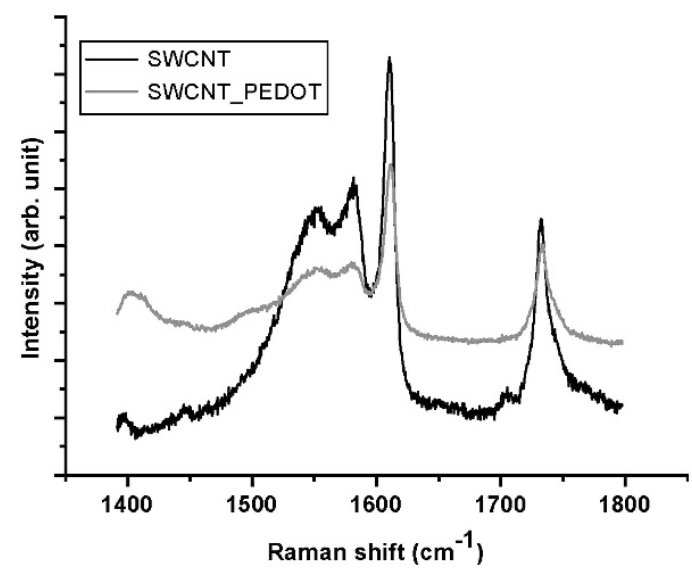

Figure 9 Raman spectra acquired at an excitation of $632.8 \mathrm{~nm}$ of the as-prepared single-walled carbon nanotubes (SWCNT) and poly $(3,4-$ ethylenedioxythiophene)-coated SWCNT networks.

was also lower. The amount of EDOT in the DBSA-EDOT complex appears to affect the reaction time and thickness of the PEDOT electropolymerized on top of the SWCNT film. This finding confirms that the thickness and sheet resistance of the PEDOT can also be controlled by changing the amount of EDOT monomer in the EDOT-DBSA complex.

\section{Raman spectroscopy examination of SWCNT and PEDOT-SWCNT networks}

Raman spectroscopy was utilized to monitor the change in the Raman peaks upon the electropolymerization of PEDOT on top of the SWCNT networks. Figure 9 shows the Raman peak positions and intensities of the SWCNT-PEDOT networks. Raman spectroscopy is a good method for confirming the doping effects in SWCNTs and graphene. A shift in the Raman position or intensity of the G- and D-bands indicates a change in the structure of the SWCNTs. It is known that the shift in the G-band peak position at approximately $1590 \mathrm{~cm}^{-1}$ is due to charge transfer, whereas the change in the Breit-Wigner-Fano shape at approximately $1545 \mathrm{~cm}^{-1}$ is due to the phonon stiffening/softening. The G-band peak shifted from 1609 to
$1611 \mathrm{~cm}^{-1}$ upon electropolymerization. A significant downshift of the G- and D-bands was observed, which is consistent with a decrease in the sheet resistance due to the PEDOT coating of the SWCNT networks. All of these changes in the Raman spectra demonstrate the effect of PEDOT on the SWCNT networks, and the creation of SWCNT hole carriers.

\section{CONCLUSION}

PEDOT was successfully electropolymerized on top of SWCNT films and exhibited a decreasing sheet resistance without sacrificing the transparency. It was possible to deposit a small amount of PEDOT on the SWCNT film or completely cover the surface by controlling the reaction time of the electropolymerization. Polymerization conditions, including the DBSA concentration, EDOT monomer concentration, voltage power and reaction time, were found to have a significant effect on the conductivity of the PEDOT-SWCNTs film. Using a specifically designed electropolymerization stage, PEDOT was electropolymerized homogeneously on top of a SWCNT film without any trade-offs between the conductivity and transparency.

\section{ACKNOWLEDGEMENTS}

This research was supported by the Gachon University Research Fund in 2011 (KWU 2011-R299).

1 Kulkarni, A. K., Schulz, K. H., Lim, T. S. \& Khan, M. Dependence of the sheet resistance of indium-tin-oxide thin film on grain size and grain orientation determined from X-ray diffraction techniques. Thin Solid Films 345, 273 (1999).

2 Chen, Z., Cotterell, B. \& Wang, W. The fracture of brittle thin films on compliant substrates in flexible displays. Eng. Fract. Mech 69, 597 (2002).

3 Lweis, B. \& Paine, D. Application and processing of transparent conducting oxides. MRS Bull 25, 22 (2000).

4 Arthur, D., Glatkowski, P., Wallis, F. \& Trottier, M. Flexible transparent circuits from carbon nanotubes. SID Int. Symp. Dig. Tec 35, 582 (2004).

5 Majumder, M., Rendall, C., Li, M., Behabtu, N., Eukel, J., Hauge, R., Schmidt, H. \& Pasquali, M. Insights into the physics of spray coating of SWNT films. Chem. Eng. Sci. 65, $2000(2010)$

6 Xiao, G., Tao, Y., Lu, J. \& Zhang, Z. Highly conductive and transparent carbon nanotube composite thin flims deposited on polythiophene terephthalate solution dipping. Thin Solid Films 518, 2822 (2010).

7 Susi, T., Kaskela, A., Zhu, Z., Ayala, P., Arenal, R., Tian, Y., Laiho, P., Mali, J., Nasibulin, A. G., Jiang, H., Lanzani, G., Stephan, O., Laasonen, K., Pichler, T., Loiseau, A. \& Kauppinen, E. I. Nitrogen-doped single-walled carbon nanotube thin films exhibiting anomalous sheet resistances. Chem. Mater. 23, 2201 (2011).

8 Williams, Q. L., Liu, X., Walters, W., Zhou, J., Edwards, T. Y. \& Smith, F. L. Boron-doped carbon nanotube coating for transparent, conducting, flexible photonic devices. Appl. Phys. Lett. 91, 143 (2007).

9 Hu, L., Choi, J. W., Yang, Y., Jeong, S., Mantia, F. L., Cui, L. \& Cui, Y. Y. Cui, P. Highly conductive paper for energy-storage devices. Natl. Acad. Sci. USA 106, 21490 (2009).

10 Tzeng, Y., Chen, Y. \& Liu, C. Diam. Electrical contacts between carbon-nanotube coated electrodes. Relat. Mater 12, 774 (2003).

11 Show, Y. \& Itabashi, H. Diam. Electrically conductive material made from CNT and PTFE. Relat. Mater 17, 602 (2008).

12 Wang, Y., Liu, H. \& Sun, X. I. Zhitomirsky, Manganese dioxide-carbon nanotube nanocomposites for electrodes of electrochemical supercapacitors. Scripta Mater. 61 1079 (2009).

13 Tang, H., Chen, J., Yao, S., Nie, L., Kuang, Y., Huang, Z., Wang, D. \& Ren, Z. Deposition and electrocatalytic properties of platinum on well-aligned carbon nanotube (CNT) arrays for methanol oxidation. Mater. Chem. Phys. 92, 548 (2005).

14 Shin, H., Jeon, S. \& Im, S. CNT/PEDOT core/shell nanostructures as a counter electrode for dye-sensitized solar cells. Synthetic Met. 161, 1284 (2011).

15 Oh, S. \& Im, S. Electroconductive polymer nanoparticles preparation and characterization of PANI and PEDOT nanoparticles. Curr. Appl. Phys. 2, 273 (2002).

16 Kim, B. H., Park, D. H., Joo, J., Yu, S. G. \& Lee, S. H. Synthesis, characteristics, and field emission of doped and de-doped polypyrrole, polyaniline, poly(3,4-ethylenedioxythiophene) nanotubes and nanowires. Synthetic Met. 150, 279 (2005).

17 Lee, H., Kim, H., Cho, M. S., Choi, J. \& Lee, Y. Fabrication of polypyrrole (PPY)/carbon nanotube (CNT) composite electrode on ceramic fabric for supercapacitor application. Electrochim. Acta 56, 7460 (2011).

18 Ou, E.C-W., Hu, L., Raymond, G. C. R., Soo, O. K., Pan, J., Zheng, Z., Park, Y., Hecht, D., Irvin, G., Drzaic, P. \& Gruner, G. Surface-modified nanotube anodes for high performance organic light-emitting diode. ACS NANO 3, 2258 (2009). 
19 Groenendaal, L. B., Jonas, F., Freitag, D., Pielartzik, H. \& Reynolds, J. R. Poly(3,4ethylenedioxythiophene) and its derivatives: past, Present, and Future. Adv. Mater. 12, 481 (2000).

20 Tantavichet, N., Pritzker, M. D. \& Burns, C. M. Electropolymerized poly(2-vinylpyridine) coatings as ion-exchange polymer modified electrodes. J. Appl. Electrochem. 31, 281 (2001).

$21 \mathrm{Kim}$, T., Kim, J., Kim, Y., Lee, T., Kim, W. \& Suh, K. S. Curr. Preparation and characterization of poly(3,4-ethylenedioxythiophene) (PEDOT) using partially sulfonated poly(styrene-butadiene-styrene) triblock copolymer as a polyelectrolyte. Appl. Phys 9, 120 (2009).

22 Chang, Y. M., Wang, L. \& Su, W. F. Polymer solar cells with poly(3,4-ethylenedioxythiophene) as transparent anode. Org. Electron. 9, 968 (2008).

23 Herzer, N., Wienk, M., Schmit, P., Spoelstra, A., Hendriks, C., Oosterhout, S., Hoeppener, S. \& Schubert, U. Fabrication of PEDOT-OTS-patterned ITO substrate. J. Mater. Chem. 20, 6618 (2010). 